\title{
LINC00839/miR-519d-3p/JMJD6 axis modulated cell viability, apoptosis, migration and invasiveness of lung cancer cells
}

\author{
Xiaoyan Yu${ }^{1 \mathrm{~A}}$, Yifei Jiang ${ }^{1 \mathrm{~B}}$, Xun $\mathrm{Hu}^{1 \mathrm{C}}$, Xiang $\mathrm{Ge}^{\mathbf{l}^{* \mathrm{D}}}$
}

The Second Affiliated Hospital of Jiaxing University, Pulmonary and Critical Care Medicine, Jiaxing, China

\begin{abstract}
Introduction. Long noncoding RNAs are associated with progressions of lung cancer. LINC00839 has been dysregulated in osteosarcoma, breast cancer and lung cancer (LC). As an upregulated lncRNA, the roles of LINC00839 in lung cancer remain unclear.

Material and methods. RNA expressions of LINC00839, miR-519d-3p and JMJD6 were assessed using RT-qPCR and JMJD6 protein expression were analyzed through Western blot. Meanwhile, viabilities of A549 and H460 LC cells transfected by siNC, siLINC00839, oeNC, oeLINC00839, NC mimics, miR-519d-3p mimics and oeLINC00839 with siJMJD6 were examined with CCK-8 assay while apoptosis was examined using flow cytometry. Meanwhile, migration and invasiveness were analyzed using transwell assays. Bindings between LINC00839 and miR-519d-3p, miR-519d-3p and JMJD6 were measured by luciferase reporter assays.

Results. LINC00839 was upregulated in LC cells and its knockdown resulted in reduced cell viability, migratory ability and invasion with increased cell apoptosis. MiR-519d-3p was the target gene of LINC00839 and its expression was reduced by LINC00839 overexpression. JMJD6 was directly targeted and suppressed at the level of mRNA and protein expression by miR-519d-3p. Moreover, miR-519d-3p overexpression resulted in low LC cell viability, migration, invasiveness but a high apoptosis rate. Furthermore, mRNA and protein expressions of JMJD6 were upregulated by LINC00839 overexpression. LINC00839 competitively sponged miR-519d-3p, increasing JMJD6 expression, LC cell viability, invasion, migratory abilities and decreasing apoptosis rates in A549 and H460 lung cancer cells, which were hindered after JMJD6 knockdown.

Conclusions. LINC00839/miR-519d-3p/JMJD6 axis mediated cell viability, apoptosis, and migration and invasiveness of H460 lung cancer cells. (Folia Histochemica et Cytobiologica 2021, Vol. 59, No. 4, 271-281)
\end{abstract}

Key words: LINC00839; miR-519d-3p; JMJD6; lung cancer

\section{Introduction}

Lung cancer (LC) is ranked as the first threating cancer worldwide, which is a global problem and a public health issue [1]. This cancer has cellular heter-

*Correspondence address: Xiang Ge,

The Second Affiliated Hospital of Jiaxing University, Pulmonary and Critical Care Medicine, No. 1518, North Huancheng Road Nanhu District, Jiaxing, Zhejiang Province, 314000. P.R. China

e-mail: ugh12254@126.com

e-mails of other authors: Artz22585@126.com;

Bdsuldehctoaf@163.com; cynuqzzyszivg@163.com;

Dugh12254@126.com ogeneity, which mainly consists of non-small cell lung cancer (NSCLC) and small cell lung cancer (SCLC) [2]. Though smoking is the major cause, pathogeneses of this malignancy become complex with the development of industry $[1,3]$. Despite progressions of surgery and molecular therapy, prognosis of lung cancer patients has been changed little [4].

Lung cancer has been demonstrated to be associated with dysregulation of noncoding RNAs [2]. Long non-coding RNAs (LncRNAs) are composed of over 200 nucleotides with no abilities to encode proteins [5], which take part in cell proliferation, differentia-

This article is available in open access under Creative Common Attribution-Non-Commercial-No Derivatives 4.0 International (CC BY-NC-ND 4.0) license, allowing to download articles and share them with others as long as they credit the authors and the publisher, but without permission to change them in any way or use them commercially. 
tion, immune reaction and so on [6-8]. LINC00839 has been determined to be an oncogene in osteosarcoma (OS) with promoting proliferation, migratory ability and invasion, in which LINC00839 sponged miR-454-3p, leading to c-Met positive regulation [9]. LINC00839 was upregulated in neuroblastoma resulting in promoted proliferation, invasion and restrained the apoptosis via miR-338-3p/GLUT1 axis in cells [10]. Reversely, knockdown of LINC00839 also hampered cell growth of neuroblastoma in xenograft models [10]. LINC00839 was found to be a differentially expressed lncRNA that was upregulated in samples of lung adenocarcinoma and lung squamous cell carcinoma [11]. MicroRNAs (miRNAs) are small endogenously expressed RNA with 20-25 nucleotides in length [2]. MiRNAs can lead to mRNA translation suppression and degradation by targeting the 3'UTR of mRNA [12]. Aberrant expressions of miRNAs also participated in the regulation of cell proliferation, differentiation and survival [2]. MiR-519d-3p was demonstrated to be down-regulated in gastric cancer tissues and it repressed proliferation and invasiveness of MGC803 cells through targeting BCL6 [13]. Beyond that, overexpression of miRNA-29c inhibited proliferation and invasiveness of NSCLC cells by downregulating VEGFA and suppressing phosphorylation of PI3K/AKT signaling pathway [14].

Jumonji domain-containing protein 6 (JMJD6) is a catalyst in lysyl hydroxylation and arginyl demethylation, which also interacts with RNA directly [15]. JMJD6 facilitated the formation of autophagosome in OS cells by phosphorylating STAT3 ${ }^{\mathrm{Y} 705 \mathrm{ph}}[16]$. Moreover, JMJD6 played an oncogenic role in NSCLC cells, promoting proliferation, migratory ability and invasiveness which were suppressed and targeted by miR-770 [17]. JMJD6 overexpression has been discovered to be related to poor prognosis and it could accelerate migration and proliferation of hepatocellular carcinoma (HCC) cells by regulating CDK4 [18]. On the contrary, knockdown of JMJD6 suppressed proliferation and survival of neuroblastoma cells in vitro and tumorigenesis in mice [19].

Competing endogenous RNAs (ceRNA) networks have been reported in cancers, in which lncRNAs could competitively sponge miRNA to regulate expressions of mRNAs [20]. Therefore, lncRNAs have been recognized as main components in the ceRNA networks contributing to the progression of tumors $[21,22]$. Putative binding sites of miR-519d-3p were offered by a bioinformatics tool, ENCORI (http://starbase.sysu.edu.cn/). Thereafter, we have proposed that LINC00839 might upregulate JMJD6 and promote the progression of lung cancer cells through sponging miR-519d-3p. To test our hypothesis, we tested cell viability, apoptosis, capacities of migration and invasion in various experimental setups with different levels of LINC00839, miR-519d-3p and JMJD6 expression.

\section{Material and methods}

Ethical statements. No animal or human samples were directly involved in this study. All the experiments were performed under the guidance of The Second OSffiliated Hospital of Jiaxing University.

Cell cultures. BEAS-2B cell line, a human normal lung epithelial cell line, was purchased from Procell (Wuhan, China) and incubated in BEGM Bullet Kit (CC-3171\&CC-4175, Lonza, Switzerland) at $37^{\circ} \mathrm{C}$ and $5 \% \mathrm{CO}_{2}$. A549 cell line, a lung cancer cell line was derived from a 58 -year-old white male and $\mathrm{H} 460$ cell line was present in hydrothorax of a male patient with non-small-cell lung cancer. Both epithelial cell lines were adherent and purchased from Procell. Ham's F-12K (PM150910, Procell) medium was used to incubate A549 cells while H460 cells were incubated in RPMI-1640 (PM150110, Procell) medium. 10\% fetal bovine serum (FBS, 164210-500, Procell) and 1\% penicillin-streptomycin solution (PB180120, Procell) were added to these two mediums and were cultured at $37^{\circ} \mathrm{C}, 5 \% \mathrm{CO}_{2}$. HEK-293 cells were also obtained from Procell for luciferase detection and cultured in MEM medium (PM150467) with 10\% FBS and $1 \%$ penicillin-streptomycin solution.

Transfection. Transfection was used to upregulate or inhibit expressions of genes. Small interfering RNAs of LINC00839 and JMJD6 and mimics of miR-519d-3p and their negative controls were provided by GenePharma (Shanghai, China). Sequences of siRNAs of LINC00839 was listed as below: siLINC00839-1, F: UAAUGACUUAUUUAACUAGAU and R: CUAGUUAAAUAAGUCAUUAUA; siLINC00839-2, F: UUAUAAUGACUUAUUUAACUA and R: GUUAAAUAAGUCAUUAUAAAU; siLINC00839-3, F: UAUGAAGAGGGUUAUUUCCAG and R: GGAAAUAACCCUCUUCAUAGU. Overexpression of LINC00839 was compounded by GeneChem (Shanghai, China). Cells $\left(1 \times 10^{5}\right.$ cells/well $)$ were cultured in 6-well plates in a volume of $2 \mathrm{ml}$ and transfection was performed after confluence of cells reached $80 \% .100$ pmol siRNAs $(20 \mu \mathrm{M}$, siLINC00839-1, siLINC00839-2, siLINC00839-3 and siJMJD6) were used for transfection and 50 pmol RNA mimics were adjusted to $25 \mu \mathrm{l}$ to prepare for transfection. Later, siRNAs, RNA mimics, oeLINC00839 and oeLINC00839 with siJMJD6 were transfected into A549 and H460 cells using Lipofectamine 3000 (Invitrogen, Carlsbad, CA, USA), respectively. Cells were incubated for another $24 \mathrm{~h}$ after transfection. RNA expressions were examined through RT-qPCR.

RT-qPCR. All RNA was isolated from BEAS-2B, A549, H460 cells and transfected A549 and H460 cells using Trizol Reagent (Invitrogen). QuantiTect Reverse Transcription 
Table 1. Sequences of primers for RT-qPCR

\begin{tabular}{|l|l|}
\hline Gene & Sequences \\
\hline LINC00839 & $\begin{array}{l}\text { Forward, 5'-CAGGTCCTGAAATCAGCCTTG-3' } \\
\text { Reverse, 5'-GCAGTTTCCACATTTGAAACCA-3' }\end{array}$ \\
\hline miR-519d-3p & $\begin{array}{l}\text { Forward, 5' - GCGGAAAAGTGCTTACAGTG -3' } \\
\text { Reverse, 5' - ATCCAGTGCAGGGTCCGAGG -3' }\end{array}$ \\
\hline JMJD6 & $\begin{array}{l}\text { Forward, 5' - TTGGAAGACTACAAGGTGCC -3' } \\
\text { Reverse, 5' - GGTCGATGTGAATCCCAGTTC -3' }\end{array}$ \\
\hline GAPDH & $\begin{array}{l}\text { Forward, 5' - ACATCGCTCAGACACCATG -3' } \\
\text { Reverse, 5' - TGTAGTTGAGGTCAATGAAGGG -3' }\end{array}$ \\
\hline U6 & $\begin{array}{l}\text { Forward, 5' - GCGGAAAAGTGCTTACAGTG -3' } \\
\text { Reverse, 5' - ATCCAGTGCAGGGTCCGAGG -3' }\end{array}$ \\
\hline
\end{tabular}

Kit (Qiagen, Dusseldorf, Germany) was applied for reverse-transcribing RNA to cDNA. Meanwhile, reverse transcription of miR-519d-3p was performed using miRCURY LNA RT Kit (Qiagen). SYBR Select Master Mix (Applied Biosystems, Carlsbad, CA, USA) and 7500 Fast PCR System (Applied Biosystems) were then applied for amplification. Sequences of primers were in Table 1 and conditions were listed: predenaturation, $95^{\circ} \mathrm{C}, 15 \mathrm{~min}$ followed by 40 cycles of denaturation, $95^{\circ} \mathrm{C}, 25 \mathrm{~s}$, annealing, $60^{\circ} \mathrm{C}, 15 \mathrm{~s}$ and extension, $72^{\circ} \mathrm{C}, 30 \mathrm{~s}$. RNA expressions were calculated by $2^{-\Delta \Delta \mathrm{Ct}}$ method and GAPDH was treated as the internal control of LINC00839 and JMJD6 and U6 was used for normalizing miR-519d-3p. This experiment was run in triplicate.

Cell viability. Viabilities of transfected A549 and H460 cells were analyzed using Cell Counting Kit-8 (Abmole Bioscience Inc, Houston, TX, USA). First, cells were cultured in a 96-well plate at a density of $5 \times 10^{3}$ cells per well in a volume of $100 \mu \mathrm{l}$. Thereafter, $10 \mu \mathrm{l} \mathrm{CCK-} 8$ was added at $24 \mathrm{~h}, 48 \mathrm{~h}$ and $72 \mathrm{~h}$ and cells were cultured for another $1 \mathrm{~h}$. Finally, absorbances (OD) of cells were detected by GloMax ${ }^{\circledR}$ Discover Microplate Reader (Promega, Madison, WI, USA). Results were collected from three independent experiments.

Cell apoptosis. Flow cytometry was applied to analyze rates of apoptosis in cancer cells. Briefly, $1 \times 10^{5}$ transfected A549 and H460 cells were resuspended in $195 \mu$ l Annexin V-FITC binding buffer (Beyotime, Shanghai, China) and then stained by $5 \mu \mathrm{l}$ Annexin V-FITC and $10 \mu \mathrm{l}$ propidium iodide (PI). Cells were cultured at $25^{\circ} \mathrm{C}$ for $10 \mathrm{~min}$ without light after staining. Apoptosis rates were then examined by BD Accuri ${ }^{\mathrm{TM}}$ C6 Plus Flow Cytometer (BD Biosciences, Franklin Lakes, NJ, USA). The experiment was repeated three times.

Dual luciferase reporter assay. After binding sites between LINC00839 with miR-519d-3p and miR-519d-3p with JMJD6 were established by using ENCORI (http://starbase. sysu.edu.cn/), bindings were confirmed by dual luciferase reporter assay. LINC00839 or JMJD6 wild type and mutant type (LINC00839-wt/mut, JMJD6-wt/mut) were first inserted into pmirGLO vectors (Promega, Madison, WI, USA), respectively. As described in the cell transfection part, Lipofectamine 3000 was applied to co-transfect vectors into HEK-293 cells $\left(1 \times 10^{5}\right.$ cells/well $)$ in a volume of $100 \mu \mathrm{l}$ with NC mimics or miR-519d-3p mimics after the cell confluence reached $70 \%$. Luciferase activities were analyzed according to the protocol of Dual-Luciferase ${ }^{\circledR}$ Reporter Assay System (Promega) after transfection for $48 \mathrm{~h}$. Experiments were run in a triplicate.

Transwell migration assay and invasion assay. Transwell assay was applied to confirm migration ability and invasiveness of transfected A549 and H460 cells. In migration detection, the upper chamber was filled with serum-free medium while the lower one was added with medium containing $10 \%$ FBS. For invasion, matrigel was coated onto the upper chamber and other conditions were the same as with transwell migration assay. Cells then were cultured for $24 \mathrm{~h}$ at $37^{\circ} \mathrm{C}, 5 \% \mathrm{CO}_{2}$. Afterward, cells in the lower chamber were stained by $1 \%$ crystal violet and numbers were counted by a microscope. The average was taken from six visual fields.

Western blot. To analyze changes of JMJD6 in protein levels, total protein was first isolated from transfected A549 and H460 cells using RIPA lysis buffer (Sigma-Aldrich, Germany) Protein was quantified using BCA protein assay kit (Thermo Scientific, MA, USA). Thereafter, 10\% SDSPAGE (Beyotime, Shanghai, China) was used for isolation of protein through electrophoresis followed by protein transferring into PVDF membranes. Primary antibodies anti-JMJD6 (1:1000, ab64575, Abcam, Cambridge, UK) and anti-GAPDH (1:2000, ab8245, Abcam) were incubated at $4{ }^{\circ} \mathrm{C}$ overnight with membranes, which were first blocked by $5 \%$ non-fat milk powder. Next, PVDF membranes were rinsed with TBST (Thermo Scientific) twice and cultured with HRP-marked goat anti-rabbit IgG (1:800, ab6721, Abcam) for $2 \mathrm{~h}$ at $25^{\circ} \mathrm{C}$. Pierce ${ }^{\mathrm{TM}}$ ECL (Thermo Scientific) was used for developing and proteins were analyzed through Image J (National Institutes of Health, USA). Experiments were run in a triplicate. 
Statistical analysis. Data were denoted as mean \pm SD and analysis of these date were conducted with SPSS 19.0 (SPSS, NY, USA) and GraphPad Prism 7 (GraphPad, CA, USA). Variances between 2 groups were analyzed through Student's $t$-test and one-way ANOVA was used for multiple groups. Significance was determined when $\mathrm{P}<0.05$. All experiments were run in a triplicate.

\section{Results}

LINC00839 was upregulated in lung cancer cell lines while downregulation of LINC00839 suppressed cell viability, migration and invasiveness but accelerated cell apoptosis.

We analyzed RNA levels of LINC00839 in lung cancer cells, indicating that LINC00839 was promoted in A549 and H460 cells versus normal BEAS-2B cells (Fig. 1A). Furthermore, LINC00839 was downregulated in A549 and H460 cells after their transfection with small interfering RNAs, and the siLINC00839-1 group had the lowest expression of LINC00839 compared to the siLINC00839-2 group and the siLINC00839-3 group (Fig. 1B). Therefore, siLINC00839-1 was selected for following experiments. Besides that, cell viabilities of A549 and H460 cells were also decreased after siLINC00839-1 transfection (Fig. 1C, D) while the apoptosis rates of cells were promoted (Fig. 1E). Moreover, knockdown of LINC00839 decreased the migration and invasiveness of A549 and H460 cells (Fig. 1F, G).

\section{LINC00839 directly targeted miR-519d-3p}

By using the bioinformatics tool (ENCORI, http:// starbase.sysu.edu.cn/), supposed binding sites between LINC00389 and miR-519d-3p were shown (Fig. 2A). Thereafter, we found that the luciferase activity was lower in the wild type of LINC00839 (LINC8839-wt) with miR-519d-3p mimics group in HEK-293 cells compared with other groups (Fig. 2B). Subsequently, we examined the miR-519d-3p RNA level, revealing that this gene was suppressed in A549 and H460 cells in comparison with normal BEAS-2B cells (Fig. 2C). Moreover, RNA expressions of miR-519d-3p were decreased in the studied LC cells after the overexpression of LINC00839 RNA (Fig. 2D).

\section{JMJD6 was targeted by miR-519d-3p}

ENCORI also provided putative binding sites between miR-519d-3p and JMJD6 (Fig. 3A). Then, luciferase reporter test revealed that the activity of fluorescence was the lowest in the miR-519d-3p with wild type of JMJD6 group (Fig. 3B). Besides that, JMJD6 RNA level was upregulated in cancer cells (Fig. 3C), which was then inhibited at both mRNA and protein levels after the transfection with miR-519d-3p mimics (Fig. $3 \mathrm{D}, \mathrm{E})$.

\section{MiR-519d-3p modulated viability, apoptosis, migratory capacity and invasiveness of lung cancer cells by suppressing JMJD6}

As JMJD6 was confirmed to be downregulated after miR-519d-3p overexpression, cell functions were analyzed next, indicating that cell viabilities of A549 and H460 cells with miR-519d-3p mimics transfection were both decreased (Fig. 4A, B). However, overexpressed miR-519d-3p promoted apoptosis rate of cancer cells (Fig. 4C). Additionally, migratory capacity and invasiveness were both reduced after cells were transfected with miR-519d-3p mimics (Fig. 4D, E).

\section{LINC00839/miR-519d-3p/JMJD6 axis modulated viability, apoptosis, invasiveness and migration capability of lung cancer cells}

Correlations among LINC00839, miR-519d-3p and JMJD6 were examined later. Results of RT-qPCR indicated that overexpressed LINC00839 promoted JMJD6 at the mRNA and protein levels which were reversed by the knockdown of JMJD6 (Fig. 5A, B). Moreover, high cell viability caused by upregulated LINC00839 was suppressed after JMJD6 inhibition (Fig. 5C). Oppositely, knockdown of JMJD6 restrained reduced apoptosis rate of A549 and H460 cells caused by the overexpression of LINC00839 (Fig. 5D). Moreover, migratory ability and invasiveness of A549 and H460 cells were both promoted after the overexpression of LINC00839 RNA, which was then decreased with knockdown of JMJD6 (Fig. 5E, F).

\section{Discussion}

Recent studies have clarified key roles of LncRNAs in progressions of tumors [23] and lncRNA has become one of the largest and multifarious RNAs in this family [24]. However, only a few of them have been demonstrated in lung cancer. LncRNA HOTAIR was promoted in lung cancer, leading to bad prognosis through promoting proliferation, invasion, migration and drug resistance, which has been demonstrated in both in vitro and clinical stages [25]. LncRNA DANCR was upregulated in tissues of NSCLC (Grade II, Grade III and Grade IV) and cells (NCI-H1299, A549 and NCI-H1975) of lung cancer which enhanced proliferation and tumor growth by sponging miR-216a [26]. In our research, we have found a new long non-coding RNA, LINC00839, which has been detected to be upregulated in lung cancer samples as 

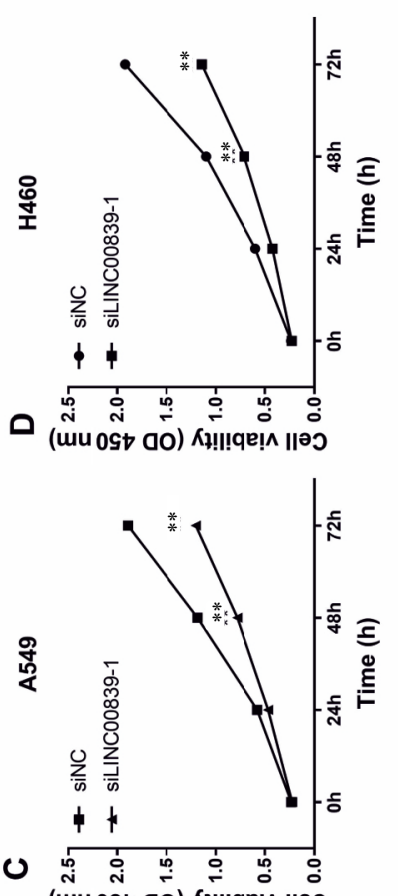

(un 0St aó) Ki!!!qe!

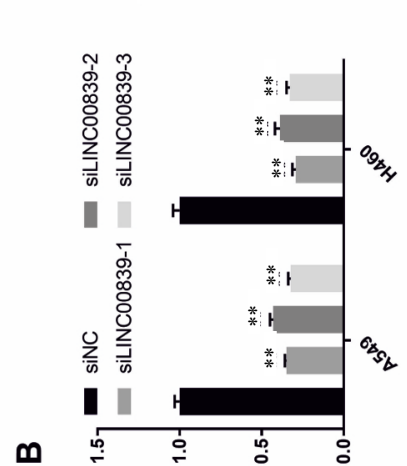

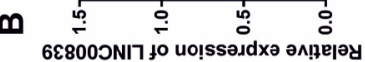

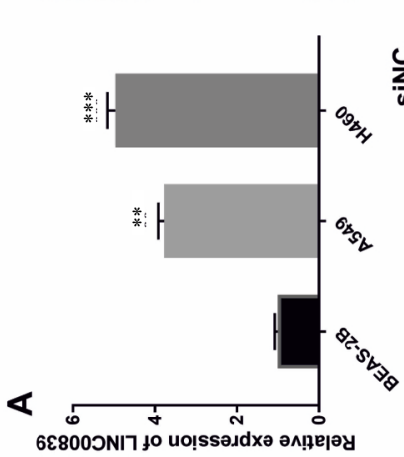

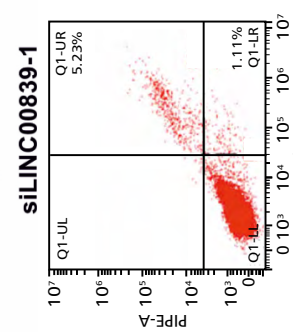

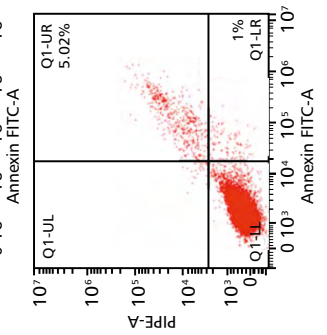

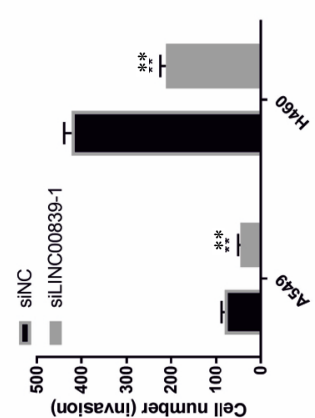
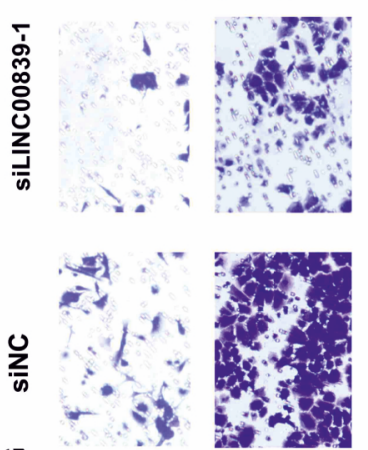

0
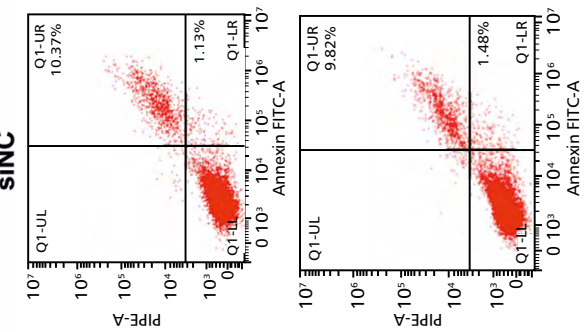

욤
守
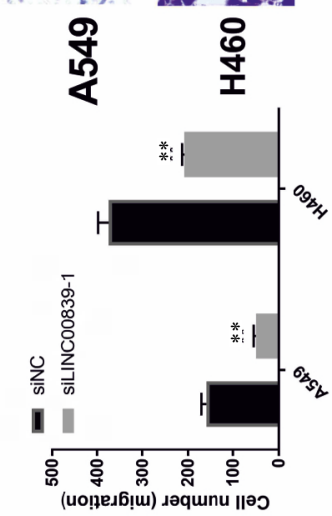

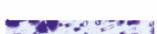

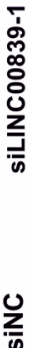

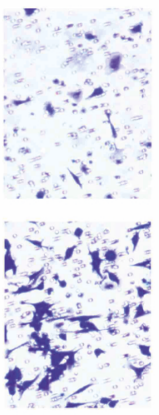

安

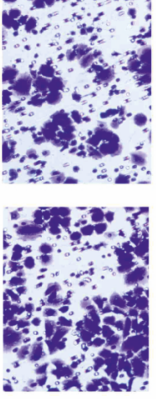

导

Figure 1. LINC00839 was upregulated in A549 and H460 cells and suppression of LINC00839 inhibited cell viability, migration and invasion but enhanced apoptosis. A. RNA expressions of LINC00839 were examined using RT-qPCR in BEAS-2B, A549 and H460 cells, ${ }^{* *} \mathrm{P}<0.05$ vs. BEAS-2B cell and ${ }^{* * *} \mathrm{P}<0.01$ vs. BEAS-2B cells. B. RNA levels of knockdown of LINC00839 (siLINC00839-1, siLINC00839-2 and siLINC00839-3) were evaluated by RT-qPCR, **P < 0.05 vs. siNC group. C, D. CCK-8 test was used for cell viability determination of A549 and H460 cells after siLINC00839-1 transfection, **P $<0.05$ in comparison with siNC group. E. Apoptosis rates of A549 and H460 cells with siLINC00839-1 transfection were evaluated using flow cytometry, ${ }^{* *} \mathrm{P}<0.05 \mathrm{vs}$. siNC group. F, G. Transwell assay was used for evaluating migratory abilities and invasiveness of A549 and H460 cells $(200 \times)$ after LINC00839 suppression, ${ }^{* *} \mathrm{P}<0.05$ in comparison with siNC group. 


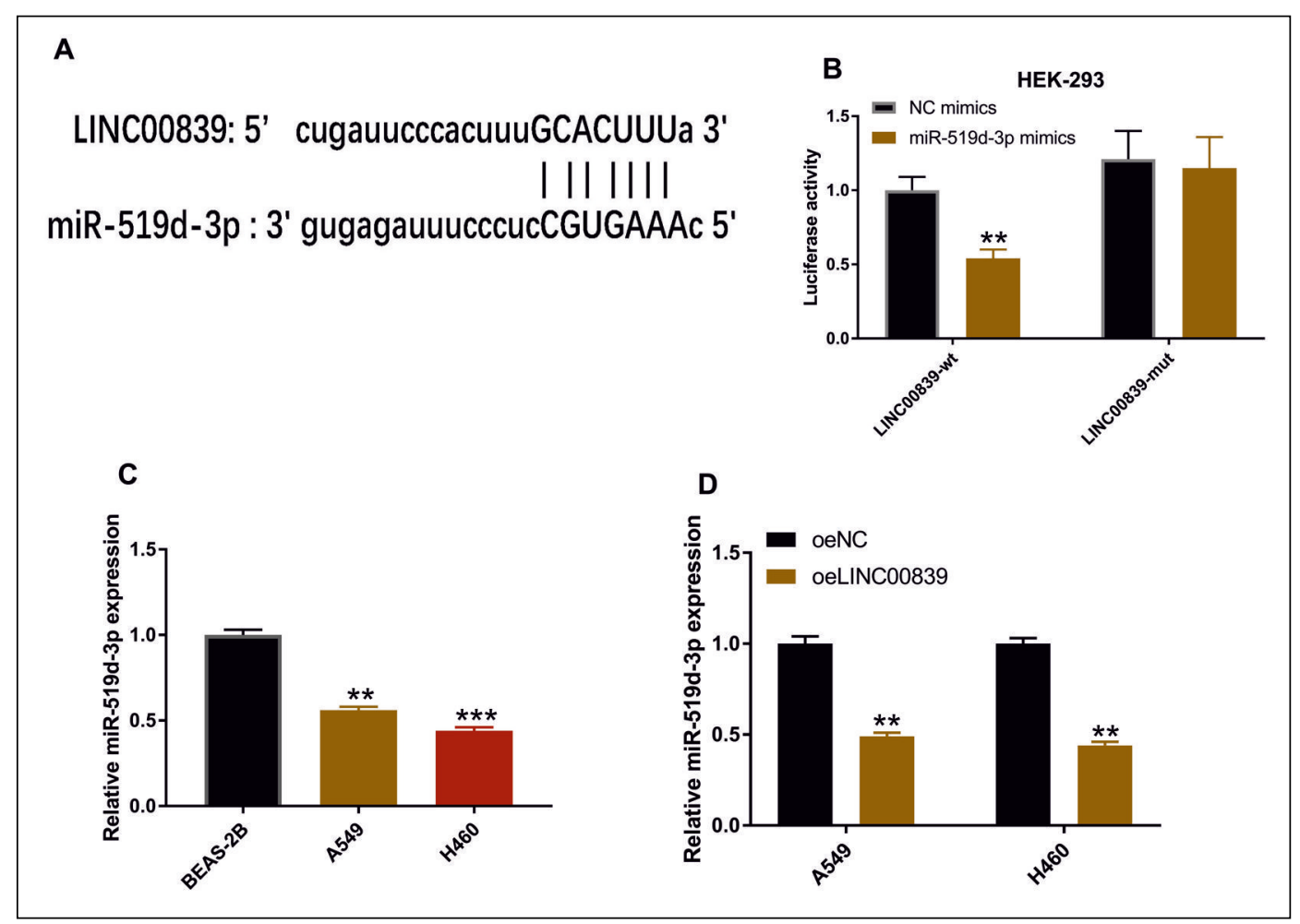

Figure 2. MiR-519d-3p was the target gene of LINC00839. A. Predicted binding sites between LINC0839 and miR-519d-3p were provided by ENCORI (http://starbase.sysu.edu.cn/). B. Luciferase reporter assay was conducted to evaluate bindings of wild type or mutant type of LINC00839 (LINC00839-wt/mut) with miR-519d-3p, ${ }^{*} \mathrm{P}<0.05$ vs. LINC00839-wt+NC mimics, LINC00839-mut + NC mimics and LINC00839-mut + miR-519d-3p mimics group. C. RT-qPCR was applied for validating RNA levels of miR-519d-3p in BEAS-2B, A549 and $\mathrm{H} 460$ cells, ${ }^{* *} \mathrm{P}<0.05$ in comparison with BEAS-2B cells and $* * * \mathrm{P}<0.01$ vs. BEAS-2B cells. D. RNA expressions of miR-519d-3p were assessed through RT-qPCR in A549 and H460 cells after the overexpression of LINC00839 RNA, **P $<0.05$ vs. oeNC group.

a differentially expressed lncRNA [11]. Nevertheless, functions of this lncRNA in lung cancer cells were still unclear. Therefore, we decided to figure out roles of this lncRNA and related mechanism. Consistently, LINC00839 was upregulated in A549 and H460 cells and knockdown of LINC00839 resulted in low cell viability, invasion and migration and in increased apoptosis. According to results of experiments, LINC00839 could facilitate biological functions of LC cells. However, these initial observations required further investigations to find out the cellular mechanisms of LINC00839 actions in LC cells.

MiRNAs have also been reported to participate in progression of lung cancer. E.g., miR-148a was decreased in tissue samples of lung cancer while its overexpression resulted in low migration and invasiveness of lung cancer cells by negatively modulating Wnt1 [27]. MiR-621 overexpression induced apoptosis of lung cancer cells by targeting SIX4, which could also improve the prognosis of patients [28]. Through bioinformatics tool (ENCORI), potential binding sites between LINC00839 and miR-519d-3p were provided and miR-519d-3p was found to be a target that was directly sponged by LINC00839. MiR-519d-3p has been discovered to retard progression of several kinds of tumors. Upregulated miR-519d-3p inhibited proliferation and arrested cell cycle of breast cancer cells (MDAMB-231 cells and HCC1937 cells) through binding LIMK1 and suppressing cofilin 1 phosphorylation [29]. MiR-519d-3p upregulation also reduced proliferation and cell cycle of prostatic cancer cells by binding TRAF4 [30]. Moreover, miR-519d-3p has been demonstrated to suppress cell proliferation, migratory ability, invasion and DDP resistance by negatively interacting with circ_007385 or HMGB1 in lung cancer cells [31]. Our study has revealed that miR-519d-3p RNA expression was also downregulated after overexpression of LINC00839 RNA. Besides that, binding sites between JMJD6 and miR-519d-3p were provided by ENCORI and the binding was confirmed using luciferase reporter assay documenting that miR-519 targeted JMJD6 directly. In the study of Zhao et al., JMJD6 was promoted by lncRNA ZFPM2-AS1, which resulted in increased proliferation and migratory capacity of breast cancer cells 


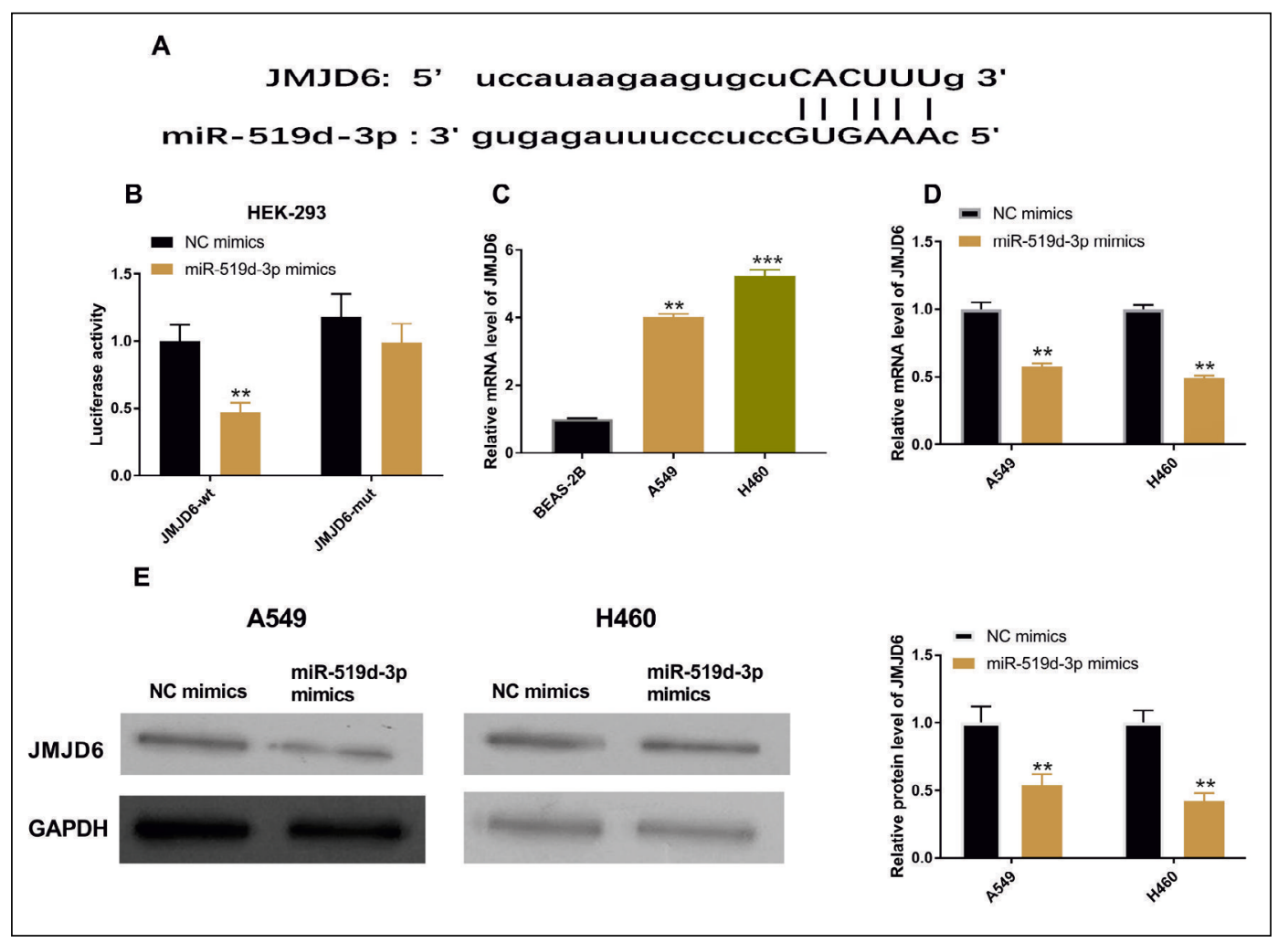

Figure 3. JMJD6, the target of miR-519d-3p, was suppressed by miR-519d-3p. A. ENCORI offered potential binding sites between miR-519d-3p and JMJD6. B. Luciferase activity of wild type of mutant type of JMJD6 (JMJD6-wt/mut) with mimics of miR-519d-3p and NC were detected by luciferase reporter assay, **P $<0.05$ compared with JMJD6-wt+NC mimics, JMJD6-mut+NC mimics and JMJD6-mut+miR-519d-3p mimics. C. RNA expressions of JMJD6 in BEAS-2B, A549 and $\mathrm{H} 460$ cells were examined using RT-qPCR, ${ }^{* *} \mathrm{P}<0.05$ in comparison with BEAS-2B cells and $* * \mathrm{P}<0.01$ vs. BEAS-2B cells. D. RT-qPCR was used to measure RNA levels of JMJD6 after upregulation of miR-519d-3p, **P $<0.05 v s$. NC mimics group. E. Protein levels of JMJD6 in A549 and H460 cells were evaluated by Western blot, **P < 0.05 vs. NC mimics group.

and increased tumor volume and weight in vivo [32]. In colon carcinogenesis, JMJD6 also facilitated proliferation and tumorigenesis in vivo and inhibited cell apoptosis in vitro by targeting p53 [33]. According to Zhang et al., relative expressions of JMJD6 at the mRNA and protein levels in lung adenocarcinoma tissues were higher than normal lung tissues, which resulted in bad prognosis [34]. JMJD6 was revealed to be an oncogene in NSCLC, which rescued low volume and weight of tumors caused by miR-770 overexpression in mice via activating the $\mathrm{Wnt} / \beta$-catenin signaling pathway [17]. Moreover, the promoter of JMJD6 gene was occupied by acetylated HOXB9 (AcK27), which suppressed migration of mouse lung cancer cells in vitro and inhibited xenografted tumor growth [35]. According to these studies, JMJD6 might be a mature biomarker that facilitated the progression of lung cancer. In this study, JMJD6 was inhibited by miR-519d-3p overexpression. Moreover, overexpressed miR-519d-3p decreased cell viability and repressed migratory and invasive capacities but enhanced apoptosis of A549 and H460 cells, which determined that miR-519d-3p might have the suppressed neoplastic potential of lung cancer cells.

Based on previous research, long non-coding RNA LCAT1 positively modulated RAC1 promoting cell proliferation and invasiveness in vitro and tumorigenesis and metastasis in vivo through competitively sponging miR-4715-5p [36]. In the study of Chen et al., IncRNA UCA1 facilitated resistance to gefitinib, accelerated cell proliferation and blocked apoptosis in NSCLC cells as a ceRNA to trigger FOSL2 through sponging miR-413 [37]. Thereafter, correlations among LINC00839, miR-519d-3p and JMJD6 were examined, in which miR-519d-3p was downregulated by LINC00839 overexpression and JMJD6 was decreased by miR-519d-3p upregulation. We have chosen overexpressed LINC00839 with suppression of JMJD6 to verify our hypothesis. After LINC00839 RNA overexpression, JMJD6 was promoted at both mRNA and protein levels while inhibition of JMJD6 reversed its expression. Additionally, enhanced cell viability, migration and invasiveness and low apoptosis of A549 and H460 cells caused by LINC00839 RNA 


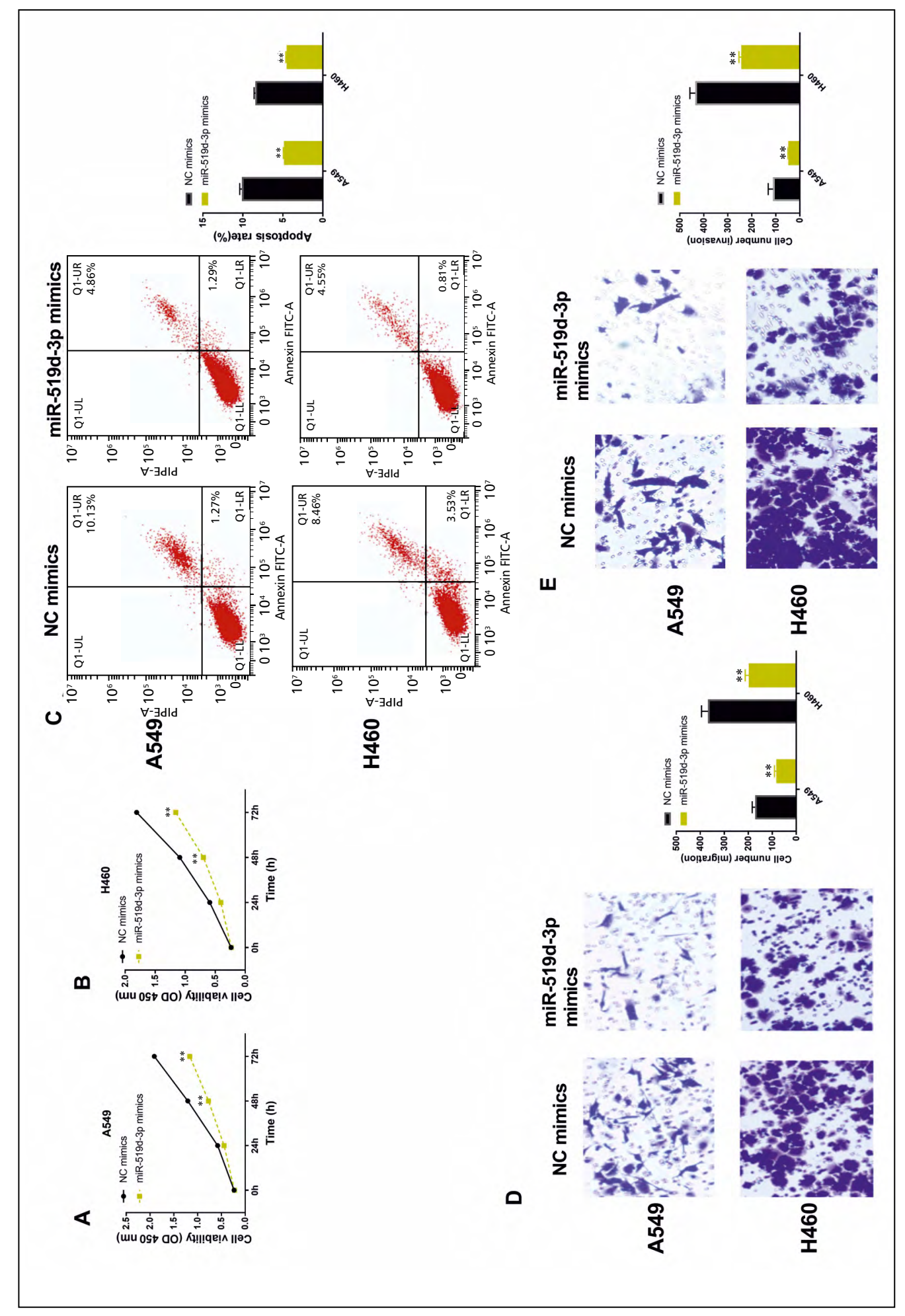

Figure 4. MiR-519d-3p modulated cell viability, apoptosis, metastasis and invasion of A549 and H460 cells through targeting JMJD6. A, B. Cell viabilities of A549 and H460 cells were analyzed using CCK-8 after miR-519d-3p overexpression, ${ }^{*} \mathrm{P}<0.05$ in comparison with $\mathrm{NC}$ mimics group. C. Flow cytometry was used to validate apoptosis rate of A549 and $\mathrm{H} 460$ cells after miR-519d-3p upregulation, ${ }^{*} \mathrm{P}<0.05 \mathrm{vs}$. NC mimics group. D, E. Migratory capacities and invasiveness of A549 and H460 cells with miR-519d-3p overexpression were checked using transwell assay $(200 \times),{ }^{* *} \mathrm{P}<0.05$ compared to NC mimics group. 


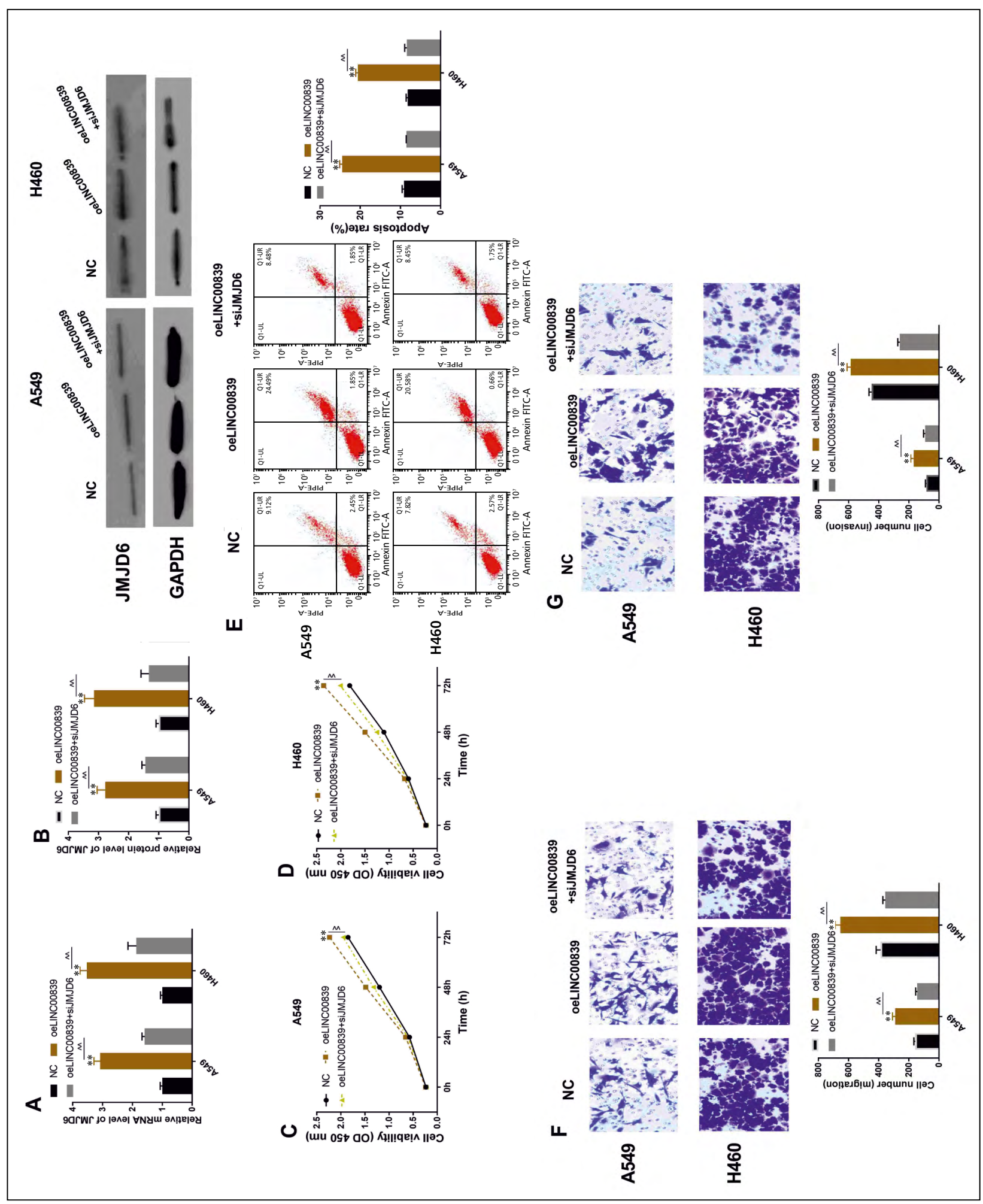

Figure 5. LINC00839/miR-519d-3p/JMJD6 axis mediated cell viability, apoptosis, migration and invasiveness of A549 and H460 cells. A, B. RNA and protein expressions of JMJD6 were assessed by RT-qPCR in A549 and H460 cells after overexpression of LINC00839 and JMJD6 inhibition, ${ }^{*} \mathrm{P}<0.05$ in comparison with NC group and $* * \mathrm{P}<0.05$ vs. oeLINC00839 group. C, D: CCK-8 was applied to measure cell viabilities of A549 and H460 cells after transfection with overexpressed LINC00839 and oeLINC00839 with siJMJD6, ${ }^{* *} \mathrm{P}<0.05$ vs. NC group and ${ }^{* *} \mathrm{P}<0.05$ in comparison with oeLINC0839 group. E. Percentage of apoptosis in A549 and H460 cells were evaluated through flow cytometry after transfecting them with oeLINC00839 and oeLINC00839 with siJMJD6, ${ }^{* *} \mathrm{P}<0.05$ vs. NC group and ${ }^{*} \mathrm{P}<0.05$ in comparison with oeLINC0839 group. F, G. Transwell assay was applied to measure abilities of A549 and H460 cell in migration as well as invasion after oeLINC00839 or oeLINC00839 with siJMJD6 transfection $(200 \times),{ }^{* *} \mathrm{P}<0.05$ vs. NC group and ${ }^{* *} \mathrm{P}<0.05$ in comparison with oeLINC0839 group. 
upregulation were decreased after JMJD6 inhibition while the repressed apoptosis was reversed to a high level. Based on these experiments, LINC00839 has been determined to upregulate JMJD6 through sponging miR-519d-3p in lung cancer cells and LINC00839/miR-519d-3p/JMJD6 axis has been verified to regulate progressions of lung cancer cells positively in vitro. Therefore, LINC00839 promoted JMJD6 accelerating cell viability, migration and invasiveness of A549 and H460 cells via competitively sponging miR-519d-3p.

In summary, LINC00839 was expressed higher in A549 and H460 cells than BEAS-2B cells and promoted cell viability, migratory and invasive abilities but repressed apoptosis as a ceRNA that triggered JMJD6 by sponging miR-519d-3p. These findings suggest that LINC00839/miR-519d-3p/JMJD6 axis components might be potential biomarkers for lung cancer treatment. However, further studies in vivo and clinical experiments are requested for a better understanding of the mechanisms of these in vitro studied phenomena.

\section{Acknowledgements}

Jiaxing City Scientific Research Project (Public Welfare Research Project) (2018AD32031).

\section{References}

1. Mao Y, Yang D, He J, et al. Epidemiology of lung cancer. Surg Oncol Clin N Am. 2016; 25(3): 439-445, doi: 10.1016/j. soc.2016.02.001, indexed in Pubmed: 27261907.

2. Ghafouri-Fard S, Shoorei H, Branicki W, et al. Non-coding RNA profile in lung cancer. Exp Mol Pathol. 2020; 114: 104411, doi: 10.1016/j.yexmp.2020.104411, indexed in $\mathrm{Pu}$ bmed: 32112788.

3. Bade BC, Dela Cruz CS. Lung cancer 2020: Epidemiology, etiology, and prevention. Clin Chest Med. 2020; 41(1): 1-24, doi: 10.1016/j.ccm.2019.10.001, indexed in Pubmed: 32008623.

4. Martin P, Leighl NB. Review of the use of pretest probability for molecular testing in non-small cell lung cancer and overview of new mutations that may affect clinical practice. Ther Adv Med Oncol. 2017; 9(6): 405-414, doi: 10.1177/1758834017704329, indexed in Pubmed: 28607579.

5. Nagano T, Fraser P. No-nonsense functions for long noncoding RNAs. Cell. 2011; 145(2): 178-181, doi: 10.1016/j. cell.2011.03.014, indexed in Pubmed: 21496640.

6. Luo $\mathrm{H}, \mathrm{Xu} \mathrm{C}, \mathrm{Le} \mathrm{W}$, et al. IncRNA CASC11 promotes cancer cell proliferation in bladder cancer through miRNA-150. J Cell Biochem. 2019; 120(8): 13487-13493, doi: 10.1002/ jcb.28622, indexed in Pubmed: 30916832.

7. Luo J, Wang K, Yeh S, et al. LncRNA-p21 alters the antiandrogen enzalutamide-induced prostate cancer neuroendocrine differentiation via modulating the EZH2/STAT3 signaling. Nat Commun. 2019; 10(1): 2571, doi: 10.1038/s41467019-09784-9, indexed in Pubmed: 31189930.

8. Robinson EK, Covarrubias S, Carpenter S. The how and why of lncRNA function: An innate immune perspective. Biochim Biophys Acta Gene Regul Mech. 2020; 1863(4): 194419, doi: 10.1016/j.bbagrm.2019.194419, indexed in Pubmed: 31487549.
9. Zhang Y, Guo H, Ma Li, et al. Long noncoding RNA LINC00839 promotes the malignant progression of osteosarcoma by competitively binding to MicroRNA-454-3p and consequently increasing c-Met expression. Cancer Manag Res. 2020; 12: 8975-8987, doi: 10.2147/CMAR.S269774, indexed in Pubmed: 33061593.

10. Yang L, Pei L, Yi J. LINC00839 regulates proliferation, migration, invasion, apoptosis and glycolysis in neuroblastoma cells through miR-338-3p/GLUT1 Axis. Neuropsychiatr Dis Treat. 2021; 17: 2027-2040, doi: 10.2147/NDT.S309467, indexed in Pubmed: 34188473.

11. Tian Y, Yu M, Sun Li, et al. Distinct patterns of mRNA and IncRNA expression differences between lung squamous cell carcinoma and adenocarcinoma. J Comput Biol. 2020; 27(7): 1067-1078, doi: 10.1089/cmb.2019.0164, indexed in Pubmed: 31750732.

12. Fabian MR, Sonenberg N, Filipowicz W. Regulation of mRNA translation and stability by microRNAs. Annu Rev Biochem. 2010; 79: 351-379, doi: 10.1146/annurev-biochem-060308-103103, indexed in Pubmed: 20533884.

13. Li YY, Shao JP, Zhang SP, et al. miR-519d-3p inhibits cell proliferation and invasion of gastric cancer by downregulating B-cell lymphoma 6. Cytogenet Genome Res. 2018; 154(1): 12-19, doi: 10.1159/000487372, indexed in Pubmed: 29510377.

14. Zhan S, Wang C, Yin F. MicroRNA-29c inhibits proliferation and promotes apoptosis in non-small cell lung cancer cells by targeting VEGFA. Mol Med Rep. 2018; 17(5): 6705-6710, doi: 10.3892/mmr.2018.8678, indexed in Pubmed: 29512752.

15. Kwok J, O'Shea M, Hume DA, et al. Jmjd6, a JmjC dioxygenase with many interaction partners and pleiotropic functions. Front Genet. 2017; 8: 32, doi: 10.3389/fgene.2017.00032, indexed in Pubmed: 28360925.

16. Wang K, Du B, Xu B, et al. JMJD6-STAT3 axis promotes autophagy in osteosarcoma cancer cells by regulating ATG. Biofactors. 2020; 46(5): 839-848, doi: 10.1002/biof.1614, indexed in Pubmed: 31958364.

17. Zhang Z, Yang Y, Zhang X. MiR-770 inhibits tumorigenesis and EMT by targeting JMJD6 and regulating WNT/ $\beta$-catenin pathway in non-small cell lung cancer. Life Sci. 2017; 188: 163-171, doi: 10.1016/j.lfs.2017.09.002, indexed in Pubmed: 28882645.

18. Wan J, Liu H, Yang L, et al. JMJD6 promotes hepatocellular carcinoma carcinogenesis by targeting CDK4. Int J Cancer. 2019; 144(10): 2489-2500, doi: 10.1002/ijc.31816, indexed in Pubmed: 30125344.

19. Wong M, Sun Y, Xi Z, et al. JMJD6 is a tumorigenic factor and therapeutic target in neuroblastoma. Nat Commun. 2019; 10(1): 3319, doi: 10.1038/s41467-019-11132-w, indexed in Pubmed: 31346162.

20. Tay Y, Rinn J, Pandolfi PP. The multilayered complexity of ceRNA crosstalk and competition. Nature. 2014; 505(7483): 344-352, doi: 10.1038/nature12986, indexed in Pubmed: 24429633.

21. Long J, Bai Yi, Yang X, et al. Construction and comprehensive analysis of a ceRNA network to reveal potential prognostic biomarkers for hepatocellular carcinoma. Cancer Cell Int. 2019; 19: 90, doi: 10.1186/s12935-019-0817-y, indexed in Pubmed: 31007608.

22. Zhou RS, Zhang EX, Sun QF, et al. Integrated analysis of IncRNA-miRNA-mRNA ceRNA network in squamous cell carcinoma of tongue. BMC Cancer. 2019; 19(1): 779, doi: 10.1186/s12885-019-5983-8, indexed in Pubmed: 31391008.

23. Wang J, Su Z, Lu S, et al. LncRNA HOXA-AS2 and its molecular mechanisms in human cancer. Clin Chim Acta. 2018; 
485: 229-233, doi: 10.1016/j.cca.2018.07.004, indexed in Pubmed: 29981289.

24. Paraskevopoulou MD, Hatzigeorgiou AG. Analyzing MiRNA-LncRNA Interactions. Methods Mol Biol. 2016; 1402: 271-286, doi: 10.1007/978-1-4939-3378-5 21, indexed in $\mathrm{Pu}$ bmed: 26721498.

25. Loewen G, Jayawickramarajah J, Zhuo Y, et al. Functions of IncRNA HOTAIR in lung cancer. J Hematol Oncol. 2014; 7: 90, doi: 10.1186/s13045-014-0090-4, indexed in Pubmed: 25491133.

26. Zhen Q, Gao LN, Wang RF, et al. LncRNA DANCR Promotes Lung Cancer by Sequestering miR-216a. Cancer Control. 2018; 25(1): 1073274818769849, doi: 10.1177/1073274818769849, indexed in Pubmed: 29651883.

27. Chen Y, Min L, Ren C, et al. miRNA-148a serves as a prognostic factor and suppresses migration and invasion through Wnt1 in non-small cell lung cancer. PLoS One. 2017; 12(2) e0171751, doi: 10.1371/journal.pone.0171751, indexed in $\mathrm{Pu}$ bmed: 28199399.

28. Zhang M, Shi H, Zhang C, et al. MiRNA-621 inhibits the malignant progression of non-small cell lung cancer via targeting SIX4. Eur Rev Med Pharmacol Sci. 2019; 23(11): 4807-4814, doi: 10.26355/eurrev_201906_18066, indexed in Pubmed: 31210312

29. Li D, Song H, Wu T, et al. MiR-519d-3p suppresses breast cancer cell growth and motility via targeting LIM domain kinase 1. Mol Cell Biochem. 2018; 444(1-2): 169-178, doi: 10.1007/s11010-017-3241-4, indexed in Pubmed: 29188531.

30. Li X, Han X, Yang J, et al. [Overexpression of miR-519d-3p inhibits the proliferation of DU-145 prostate cancer cells by reducing TRAF4]. Xi Bao Yu Fen Zi Mian Yi Xue Za Zhi. 2018; 34(1): 16-21, indexed in Pubmed: 29595452.

31. Ye Y, Zhao L, Li Q, et al. circ 0007385 served as competing endogenous RNA for miR-519d-3p to suppress malignant behaviors and cisplatin resistance of non-small cell lung cancer cells. Thorac Cancer. 2020; 11(8): 2196-2208, doi: 10.1111/1759-7714.13527, indexed in Pubmed: 32602212.

32. Zhao YF, Li L, Li HJ, et al. LncRNA ZFPM2-AS1 aggravates the malignant development of breast cancer via upregulating JMJD6. Eur Rev Med Pharmacol Sci. 2020; 24(21): 11139-11147, doi: 10.26355/eurrev_202011_23601, indexed in Pubmed: 33215431.

33. Wang F, He L, Huangyang P, et al. JMJD6 promotes colon carcinogenesis through negative regulation of $\mathrm{p} 53$ by hydroxylation. PLoS Biol. 2014; 12(3): e1001819, doi: 10.1371/journal. pbio.1001819, indexed in Pubmed: 24667498.

34. Zhang Ji, Ni SS, Zhao WL, et al. High expression of JMJD6 predicts unfavorable survival in lung adenocarcinoma. Tumour Biol. 2013; 34(4): 2397-2401, doi: 10.1007/s13277-0130789-9, indexed in Pubmed: 23595221.

35. Wan J, Xu W, Zhan J, et al. PCAF-mediated acetylation of transcriptional factor HOXB9 suppresses lung adenocarcinoma progression by targeting oncogenic protein JMJD6. Nucleic Acids Res. 2016; 44(22): 10662-10675, doi: 10.1093/ nar/gkw808, indexed in Pubmed: 27613418.

36. Yang J, Qiu Q, Qian X, et al. Long noncoding RNA LCAT1 functions as a ceRNA to regulate RAC1 function by sponging miR-4715-5p in lung cancer. Mol Cancer. 2019; 18(1): 171, doi: 10.1186/s12943-019-1107-y, indexed in Pubmed: 31779616

37. Chen X, Wang Z, Tong F, et al. IncRNA UCA1 promotes gefitinib resistance as a ceRNA to target FOSL2 by sponging miR-143 in non-small cell lung cancer. Mol Ther Nucleic Acids. 2020; 19: 643-653, doi: 10.1016/j.omtn.2019.10.047, indexed in Pubmed: 31951852.

Submitted: 21 July, 2021

Accepted after reviews: 20 October, 2021 Available as AoP: 4 November, 2021 